\title{
Synthesis and antimicrobial activity evaluation of novel nitrofuranthiazoles
}

\author{
Leyla Yurttaş,", Zafer Şahin², Sevda Er ${ }^{3}$, Şeref Demirayak² \\ ${ }^{1}$ Anadolu University, Faculty of Pharmacy, Department of Pharmaceutical Chemistry, 26470, Eskişehir, Turkey \\ 2Medipol University, School of Pharmacy, Department of Pharmaceutical Chemistry, 34810, İstanbul, Turkey \\ ${ }^{3}$ Medipol University, School of Pharmacy, Department of Pharmaceutical Microbiology, 34810, İstanbul, Turkey
}

\begin{abstract}
In this work, six novel 4-aryl-2-[2-((5-nitrofuran-2-yl)methylene)hydrazinyl] thiazole derivatives (2a-f) were synthesized starting from 5-nitro-2-furaldehyde diacetate by using Hantzsch thiazole synthesis. The antimicrobial activity of the title compounds were screened against five Gram positive bacteria B. cereus, E. faecalis, $S$. aureus, $S$. epidermidis, $L$. monocytogenes and two Gram negative bacteria $E$. coli and $S$. typhi. MIC and MBC were calculated and compared to standard drug nitrofurazone. Compounds bearing pyridine moiety (2d-e) exhibited significant antimicrobial activity which could be evaluated as new, potent antibacterial agents.
\end{abstract}

Keywords: nitrofurans, nitrofurazone, thiazole, antibacterial activity.

\section{INTRODUCTION}

The incidence of microbial infections have been increasing day by day in worldwide. Many antibacterial agents are in use against a wide range of infectious diseases $^{1,2}$. However, the resistance to existing drugs is still a serious problem threat to global public health. This situation leads medicinal chemists to investigate newly synthesized more potent antimicrobial drugs ${ }^{3}$. Among many antibacterial agents, nitrofuran containing drugs (Figure 1. furazolidone, nitrofurantoin nitrofurazone and furaltadone) have been widely used for protection against microbial and protozoal infections especially associated with food contaminations ${ }^{4}$. The nitrofurans are characterized by a 5 -nitro-2-furanyl group. First synthesized drug nitrofurazone, 5-nitro-2- furaldehyde semicarbazone, is still being used molecule due to its antitrypanosomal activity as well as antibac-

*Corresponding author: Leyla Yurttaş

E-mail address: lyurttas@anadolu.edu.tr 
terial potential to treat burns topically5. However, mutagenic/carcinogenic toxic effects of this molecule were detected for the antimicrobial and anti-protozoal applications ${ }^{6}$. Therefore, prodrug approach was suggested to increase biological activity and decrease toxicity along with improving the physico-chemical properties $^{7}$. For this purpose, many 5 -nitrofuryl derivatives have been extensively studied for the treatment of various microbial infections ${ }^{8-12}$ and these compounds are determined to act via producing oxidative stress on the parasite which leads to death of the microbe ${ }^{13}$.

Addition to all, nitrofuran agents have been developed through combining thiosemicarbazone moiety and different heterocyclic rings. Thiosemicarbazones and their derivatives are important compounds known with many biological properties especially as chemotherapeutic agents ${ }^{14-18}$. Their cyclization products, (4-aryl-thiazol-2-yl)hydrazines are also widely studied derivatives due to their numerous pharmacological applications and varied biological activities ${ }^{19-21}$. Also, thiazole containing compounds have attracted broad interest because of their synthesis ease of reaction and their capability to easily furnish valuable chemotherapeutics such as anticancer, antibacterial, antifungal, and antiprotozoal agents $^{22}$.

Considering the reported data, we have described six novel compounds combining (4-aryl-thiazol-2-yl)hydrazine and 5-nitrofuryl moieties which we based on well-known antibacterial agent, nitrofurazone. The antibacterial activity of the compounds have been investigated against various microorganisms compared with standard drug.

\section{METHODOLOGY}

\section{Chemistry}

Melting points were determined by MP9o digital melting point apparatus (Mettler Toledo, $\mathrm{OH}$ ) and were uncorrected. Spectroscopic data were obtained by instruments: Bruker Tensor 27 IR spectrophotometer; ${ }^{1} \mathrm{H}$ NMR (nuclear magnetic resonance) Bruker DPX- 300 FT-NMR spectrometer, ${ }^{13} \mathrm{C}$ NMR, Bruker DPX 75 MHz spectrometer (Bruker Bioscience, Billerica, MA, USA); M+1 peaks were determined by Shimadzu LC/MS ITTOF system (Shimadzu, Tokyo, Japan).

\section{Preparation of 5-nitrofuran-2-carbaldehyde thiosemicarbazone (1)}

A mixture of thiosemicarbazide (0.27 g, $3 \mathrm{mmol}$ ) and 5-nitro-2-furaldehyde diacetate $(3 \mathrm{mmol})$ in ethanol $(20 \mathrm{~mL})$ was refluxed for $3 \mathrm{~h}$. After keeping the solution at $\mathrm{O}^{\circ} \mathrm{C}$ overnight, the precipitated raw product was filtered off and recrystallized from ethanol to afford corresponding thiosemicarbazone compound (1). 


\section{Preparation of 4-aryl-2-[2-((5-nitrofuran-2-yl)methylene) hydrazinyl]thiazole derivatives (2a-f)}

5-Nitro-2-furaldehyde thiosemicarbazone (1) (0.2 $\mathrm{mmol})$ and appropriate a-bromoarylethanone derivative ( $0.16 \mathrm{~g}, 0.80 \mathrm{mmol})$ were stirred in ethanol at room temperature. The progress of the reaction was monitored by TLC. The mixture was filtered, the product was dried and recrystallized from ethanol, to give target compounds (2a-f).

\section{4-(2-Hydroxyphenyl)-2-[2-((5-nitrofuran-2-yl)methylene) hydrazinyl]thiazole (2a)}

$72 \%$ yield; mp $221^{\circ} \mathrm{C}$. IR $v_{\max }\left(\mathrm{cm}^{-1}\right): 3452(\mathrm{OH}), 3121$ (amide $\left.\mathrm{N}-\mathrm{H}\right), 1574$ and $1359\left(\mathrm{NO}_{2}\right), 1479-1454(\mathrm{C}=\mathrm{C}, \mathrm{C}=\mathrm{N}), 1171-1014(\mathrm{C}-\mathrm{O}, \mathrm{C}-\mathrm{N}) .{ }^{1} \mathrm{H}-\mathrm{NMR}$ (300 MHz, DMSO- $d_{6}$, ppm) $\delta$ 6.84-6.92 (m, 2H, Ar-H), 7.13-7.19 (m, 2H, Ar-H), 7.52 (s, $1 \mathrm{H}$, thiazole $\mathrm{C}_{5}-\mathrm{H}$ ), 7.79 (d, $\left.J=3.93 \mathrm{~Hz}, 1 \mathrm{H}, \mathrm{Ar}-\mathrm{H}\right), 7.85$ (d, $J=7.77 \mathrm{~Hz}, 1 \mathrm{H}, \mathrm{Ar}-\mathrm{H}$ ), 7.99 (s, $1 \mathrm{H},-\mathrm{CH}=\mathrm{N}$ ), 10.84 (brs, $1 \mathrm{H}, \mathrm{OH}), 12.79$ (brs, $1 \mathrm{H}, \mathrm{NH}) .{ }^{13} \mathrm{C}-\mathrm{NMR}(75 \mathrm{MHz}$, DMSO- $d_{6}$, ppm) $\delta$ 106.54, 114.49, 115.58, 117.06, 119.63, 127.94, 129.40, 129.86, 152.08, 152.42, 155.53. HRMS (m/z): $[\mathrm{M}+\mathrm{H}]^{+}$calcd for $\mathrm{C}_{14} \mathrm{H}_{10} \mathrm{~N}_{4} \mathrm{O}_{4} \mathrm{~S} 331.32$; found 331.05 .

\section{4-(3-Hydroxyphenyl)-2-[2-((5-nitrofuran-2-yl)methylene) hydrazinyl]thiazole (2b)}

$70 \%$ yield; mp $188^{\circ} \mathrm{C}$. IR $v_{\max }\left(\mathrm{cm}^{-1}\right): 3450(\mathrm{OH}), 3112$ (amide $\left.\mathrm{N}-\mathrm{H}\right), 1566$ and $1315\left(\mathrm{NO}_{2}\right), 1512-1385(\mathrm{C}=\mathrm{C}, \mathrm{C}=\mathrm{N}), 1198-1016(\mathrm{C}-\mathrm{O}, \mathrm{C}-\mathrm{N}) .{ }^{1} \mathrm{H}-\mathrm{NMR}$ (300 MHz, DMSO- $d_{6}$ ppm) $\delta$ 6.91-6.74 (m, 1H, Ar-H), $7.13(\mathrm{~d}, J=3.991 \mathrm{H}, \mathrm{Ar}-\mathrm{H}), 7.21$ (t, $J=7.89 \mathrm{~Hz}, 1 \mathrm{H}, \mathrm{Ar}-\mathrm{H}), 7.28-7.33\left(\mathrm{~m}, 3 \mathrm{H}\right.$, thiazole $\mathrm{C}_{5}-\mathrm{H}$ and $\left.\mathrm{Ar}-\mathrm{H}\right), 7.78(\mathrm{~d}$, $J=3.93 \mathrm{~Hz}, 1 \mathrm{H}, \mathrm{Ar}-\mathrm{H}$ ), 7.98 (s, $1 \mathrm{H},-\mathrm{CH}=\mathrm{N}$ ), 9.49 (brs, $1 \mathrm{H}, \mathrm{OH}), 12.75$ (brs, $1 \mathrm{H}$, $\mathrm{NH}) .{ }^{13} \mathrm{C}-\mathrm{NMR}$ (75 MHz, DMSO- $d_{6}$ ppm) $\delta$ 105.22, 112.98, 114.19, 115.26, 116.88, $117.25,129.31$, 130.07, 130.98, 136.07, 151.22, 152.02, 152.59, 158.04, 167.42. HRMS (m/z): $[\mathrm{M}+\mathrm{H}]^{+}$calcd for $\mathrm{C}_{14} \mathrm{H}_{10} \mathrm{~N}_{4} \mathrm{O}_{4} \mathrm{~S} 331.32$; found 331.05.

\section{4-(4-Hydroxyphenyl)-2-[2-((5-nitrofuran-2-yl)methylene) hydrazinyl]thiazole (2c)}

$76 \%$ yield; mp $210{ }^{\circ} \mathrm{C}$. IR $v_{\max }\left(\mathrm{cm}^{-1}\right): 3454(\mathrm{OH}), 3120$ (amide $\mathrm{N}-\mathrm{H}$ ), 1573 and $1317\left(\mathrm{NO}_{2}\right), 1471-1317(\mathrm{C}=\mathrm{C}, \mathrm{C}=\mathrm{N}), 1201-1012(\mathrm{C}-\mathrm{N}) .{ }^{1} \mathrm{H}-\mathrm{NMR}$ (300 MHz, DMSO- $d_{6}$, ppm) $\delta 6.81(\mathrm{~d}, J=8.64 \mathrm{~Hz}, 2 \mathrm{H}, \mathrm{Ar}-\mathrm{H}), 7.11-7.14(\mathrm{~m}, 2 \mathrm{H}$, thiazole $\mathrm{C}_{5}-\mathrm{H}$ and $\mathrm{Ar}-\mathrm{H}$ ), 7.68 (d, $\left.J=8.61 \mathrm{~Hz}, 2 \mathrm{H}, \mathrm{Ar}-\mathrm{H}\right), 7.77$ (d, $\left.J=3.96 \mathrm{~Hz}, 1 \mathrm{H}, \mathrm{Ar}-\mathrm{H}\right)$, $7.96(\mathrm{~s}, 1 \mathrm{H},-\mathrm{CH}=\mathrm{N}), 9.58$ (s, $1 \mathrm{H}, \mathrm{OH}), 12.7 \mathrm{O}$ (brs, $1 \mathrm{H}, \mathrm{NH}) .{ }^{13} \mathrm{C}-\mathrm{NMR}(75 \mathrm{MHz}$, DMSO- $d_{6,}$ ppm) $\delta$ 102.26, 114.09, 115.64, 115.82, 126.13, 127.42, 129.18, 151.99, 152.68, 157.68, 167.43. HRMS (m/z): $[\mathrm{M}+\mathrm{H}]^{+}$calcd for $\mathrm{C}_{14} \mathrm{H}_{10} \mathrm{~N}_{4} \mathrm{O}_{4} \mathrm{~S} 331.32$; found 331.05 . 


\section{4-(2-Pyridyl)-2-[2-((5-nitrofuran-2-yl)methylene)hydrazinyl] thiazole (2d)}

$76 \%$ yield; mp $230{ }^{\circ} \mathrm{C}$. IR $v_{\max }\left(\mathrm{cm}^{-1}\right): 3113$ (amide $\mathrm{N}-\mathrm{H}$ ), 1516 and $1348\left(\mathrm{NO}_{2}\right)$, 1471-1313 (C=C, C=N), 1298-1024 (C-N, C-O). ${ }^{1} \mathrm{H}-\mathrm{NMR}$ (300 MHz, DMSO-d ppm) $\delta 7.19$ (d, $J=3.96 \mathrm{~Hz}, 1 \mathrm{H}, \mathrm{Ar}-\mathrm{H}), 7.72-7.79$ (m, 2H, Ar-H), 8.10 (d, $J=4.14$ $\mathrm{Hz}, 2 \mathrm{H}, \mathrm{Ar}-\mathrm{H}$ ), 8.29 (d, J=7.89 Hz, $1 \mathrm{H}, \mathrm{Ar}-\mathrm{H}$ ), 8.38 (t, $J=8.76 \mathrm{~Hz}, 1 \mathrm{H}, \mathrm{Ar}-\mathrm{H}$ ), 8.72 (d, $J=4.74 \mathrm{~Hz}, 1 \mathrm{H}, \mathrm{Ar}-\mathrm{H}), 11.85$ (brs, $1 \mathrm{H}, \mathrm{NH}) .{ }^{13} \mathrm{C}-\mathrm{NMR}\left(75 \mathrm{MHz}, \mathrm{DMSO}-d_{6}\right.$, ppm) $\delta$ 114.56, 114.95, 114.91, 115.48, 123.08, 123.85, 124.98, 130.76, 143.97, 144.84, 145.48, 147.62, 152.09, 152.16, 168.74. HRMS (m/z): $[\mathrm{M}+\mathrm{H}]^{+}$calcd for $\mathrm{C}_{13} \mathrm{H}_{9} \mathrm{~N}_{5} \mathrm{O}_{3} \mathrm{~S} 316.31$; found 316.05 .

\section{4-(3-Pyridyl)-2-[2-((5-nitrofuran-2-yl)methylene)hydrazinyl] thiazole (2e)}

$71 \%$ yield; mp $240{ }^{\circ} \mathrm{C}$. IR $v_{\max }\left(\mathrm{cm}^{-1}\right): 3115$ (amide $\left.\mathrm{N}-\mathrm{H}\right), 1568$ and $1352\left(\mathrm{NO}_{2}\right)$, 1475-1315 (C=C, C=N), 1302-1016 (C-N, C-O). ${ }^{1} \mathrm{H}-\mathrm{NMR}$ (300 MHz, DMSO- $d_{6}$, ppm) $\delta$ 7.77-7.79 (m, 1H, Ar-H), 8.39-8.48 (m, 3H, Ar-H), 8.64 (s, 1H, thiazole $\left.\mathrm{C}_{5}-\mathrm{H}\right), 9.23-9.34(\mathrm{~m}, 2 \mathrm{H}, \mathrm{Ar}-\mathrm{H}), 9.80(\mathrm{~s}, 1 \mathrm{H},-\mathrm{CH}=\mathrm{N}), 13.50$ (brs, $\left.1 \mathrm{H}, \mathrm{NH}\right)$. ${ }^{13} \mathrm{C}-\mathrm{NMR}\left(75 \mathrm{MHz}, \mathrm{DMSO}-d_{6}\right.$ ppm) $\delta$ 110.11, 115.33, 116.16, 126.95, 130.73, 132.92, 138.79, 143.39, 144.99, 147.00, 152.74, 152.88, 169.11. HRMS ( $\mathrm{m} / \mathrm{z})$ : $[\mathrm{M}+\mathrm{H}]^{+}$calcd for $\mathrm{C}_{13} \mathrm{H}_{9} \mathrm{~N}_{5} \mathrm{O}_{3} \mathrm{~S} 316.31$; found 316.05 .

\section{4-(4-Pyridyl)-2-[2-((5-nitrofuran-2-yl)methylene)hydrazinyl] thiazole (2f)}

$69 \%$ yield; mp $236{ }^{\circ} \mathrm{C}$. IR $v_{\max }\left(\mathrm{cm}^{-1}\right): 3115$ (amide $\left.\mathrm{N}-\mathrm{H}\right), 1556$ and $1346\left(\mathrm{NO}_{2}\right)$, 1481-1323 (C=C, C=N), 1247-1012 (C-N, C-O). ${ }^{1} \mathrm{H}-\mathrm{NMR}$ (300 MHz, DMSO- $d_{6}$, ppm) $\delta 7.18$ (d, $J=\mathrm{Hz}, 1 \mathrm{H}, \mathrm{Ar}-\mathrm{H}$ ), 7.78 (d, $J=\mathrm{Hz}, 1 \mathrm{H}, \mathrm{Ar}-\mathrm{H}), 8.03$ (s, $1 \mathrm{H}$, thiazole $\left.\mathrm{C}_{5}-\mathrm{H}\right), 8.27-8.30(\mathrm{~m}, 3 \mathrm{H},-\mathrm{CH}=\mathrm{N}$ and $\mathrm{Ar}-\mathrm{H}), 8.86$ (d, $\left.J=6.40 \mathrm{~Hz}, 2 \mathrm{H}, \mathrm{Ar}-\mathrm{H}\right)$, 12.96 (brs, $1 \mathrm{H}, \mathrm{NH}) .{ }^{13} \mathrm{C}-\mathrm{NMR}$ (75 MHz, DMSO- $d_{6}$ ppm) $\delta 114.96,115.48,116.30$, 122.23, 130.56, 144.11, 146.82, 147.95, 152.08, 147.00, 152.16, 168.62. HRMS $(\mathrm{m} / \mathrm{z}):[\mathrm{M}+\mathrm{H}]^{+}$calcd for $\mathrm{C}_{13} \mathrm{H}_{9} \mathrm{~N}_{5} \mathrm{O}_{3} \mathrm{~S} 316.31$; found 316.05 .

\section{Antibacterial activity}

Antibacterial activity against Bacillus cereus ATCC 14579, Escherichia coli ATCC 25922, Enterococcus faecalis ATCC 29212, Staphylococcus aureus ATCC 25923, Staphylococcus epidermidis ATCC 12228, Salmonella typhi ATCC 14028, and Listeria monocytogenes ATCC 7644 was determined by the microbroth dilutions technique using the Clinical Laboratory Standarts Institute (CLSI) recommendations ${ }^{23}$.

The minimum inhibitory concentrations (MIC) was defined as the lowest concentration of compound giving complete inhibition of visible growth and the 
minimum bactericidal concentration (MBC) was defined as the lowest concentration of the compound to completely kill bacteria. Nitrofurazone was used as positive control. According to values of the controls, the results were evaluated. Each experiment was replicated twice.

\section{RESULTS AND DISCUSSION}

\section{Chemistry}

In this study, 4-aryl-2-[2-((5-nitrofuran-2-yl)methylene)hydrazinyl]thiazole derivatives (2a-f) were synthesized with a two-step synthetic procedure as shown Scheme 1. Compound 1 was synthesized by the reaction of 5-nitro-2-furaldehyde diacetate and thiosemicarbazide in ethanol at reflux conditions. The gained 5-nitrofuran-2-carbaldehyde thiosemicarbazone is a previously reported molecule with a melting point of $163-165^{\circ} \mathrm{C}^{24}$. In second step, six new derivatives (2af) were synthesized via Hantzsch thiazole synthesis. 2-/3- or /4-Hydroxyphenyla-bromoethanone and 2-/3- or 4-pyridyl-a-bromoethanones were used as $\alpha$-haloketone compounds. In this way, six novel 4-aryl-2-[2-((5-nitrofuran-2-yl) methylene)hydrazinyl]thiazole derivatives (2a-f) were obtained with the 68-76 $\%$ yield.

Characteristic infra-red stretching bands belong to amine (NH) and nitro $\left(\mathrm{NO}_{2}\right)$ groups were detected at $3121-3112 \mathrm{~cm}^{-1}$ and about $1550,1340 \mathrm{~cm}^{-1}$, respectively. In ${ }^{1} \mathrm{H}$ NMR spectra, peaks of azomethine $(-\mathrm{CH}=\mathrm{N})$ and amine protons were detected in range 7.99-7.89 ppm and 11.85-12.89 ppm, respectively. OH protons of 2a-c were detected at about 9.49-10.84 ppm as broad singlet peaks. In the ${ }^{13} \mathrm{C}$ NMR spectra, all carbons were observed at 102.26-169.11 ppm range, correctly. Molecular ion peaks were also determined in agreement with molecular weights of the compounds.

\section{Biology}

Final compounds (2a-f) were screened to determine their antimicrobial activities against totally seven Gram negative and Gram positive bacteria; E. coli, $S$. typhi, E. faecalis, S. aureus, S. epidermidis, L. monocytogenes and B. cereus. MIC and MBC were identified for standart drug nitrofurazone and all compounds. As were represented in Table 1, The MIC and MBC values were found between $0.5-2 \mathrm{mg} / \mathrm{mL}$ and higher for the 2a-c, whereas both of the values were found as $1 \mathrm{mg} / \mathrm{mL}$ and higher for nitrofurazone.

Among the hydroxylated compounds (2a-c), compound $2 \mathbf{a}$ exhibited the highest activity against $B$. cereus ( $\mathrm{MIC}=0.5 \mathrm{mg} / \mathrm{mL}, \mathrm{MBC}=1 \mathrm{mg} / \mathrm{mL}$ ). Besides, it showed same potency to nitrofurazone (MIC: $1 \mathrm{mg} / \mathrm{mL}$ ) against $E$. coli and $E$. faecalis. Compound $\mathbf{2 b}$ did not show prominent antibacterial activity which had 
MIC and MBC values $2 \mathrm{mg} / \mathrm{mL}$ and higher against all tested bacteria. Another hydroxyl containing compound $\mathbf{2 c}$ showed same antibacterial potency to standard drug against three bacteria, $S$. aureus, S. typhi and L. monocytogenes.

Regarding to compounds 2d-e with pyridine moiety, they exhibited significant activity with a higher potency than hydroxylated compounds (2a-c). Compound 2d including 2-pyridyl moiety showed two-fold antibacterial potency against B. cereus (MIC and MBC: $0.5 \mathrm{mg} / \mathrm{mL}$ ) compared to nitrofurazone. It exhbited same potency to standard drug against E. coli and S. epidermis. Also, the lowest concentrations of compound $\mathbf{2 d}$ caused inhibition growth of bacteria (MIC) were found as $1 \mathrm{mg} / \mathrm{mL}$ whereas the lowest concentrations of the compound caused completely death of bacteria (MBC) were found higher than the highest tested concentration (>2 mg/mL) against E. faecalis, S. aureus, S. typhi and L. monocytogenes. MIC values for compound 2 e were found between 0.5-1 mg/ $\mathrm{mL}$ whereas $\mathrm{MBC}$ values were found as $2 \mathrm{mg} / \mathrm{mL}$. Additionally, compound $\mathbf{2 e}$ containing 3-pyridine moiety could be declared as the most active compound with the lowest MIC values among the other compounds. Also, sixth compound 2f bearing 4-pyridyl moiety showed remarkable activity. MIC and MB values were calculated as $0.5 \mathrm{mg} / \mathrm{mL}$ against the most susceptible bacteri B. cereus.<smiles>O=C1OCCN1/N=C/c1ccc([N+](=O)[O-])o1</smiles><smiles>O=C1CN(/N=C/c2ccc([N+](=O)[O-])o2)C(=O)N1</smiles><smiles>NC(=O)N/N=C/c1ccc([N+](=O)[O-])o1</smiles>

(c)<smiles>O=C1OC(CN2CCOCC2)CN1/N=C/c1ccc([N+](=O)[O-])o1</smiles>

Figure 1: Nitrofuran drugs. (a) Furazolidone, (b) Nitrofurantoin, (c) Nitrofurazone, (d) Furaltadone.

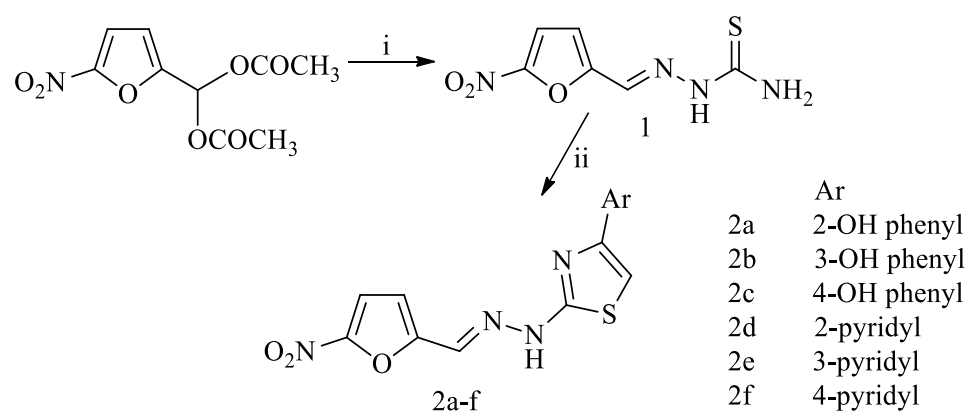

Scheme 1: Synthesis of the compounds (2a-f). Reagents: (i) thiosemicarbazide, ethanol, reflux, 3h; (ii) $\alpha$-bromoarylethanone, ethanol, rt. 
Table 1: Antimicrobial activity of the compounds $(\mathrm{mg} / \mathrm{mL})$

\begin{tabular}{|c|c|c|c|c|c|c|c|c|}
\hline \multicolumn{2}{|c|}{} & \multicolumn{7}{|c|}{ Compounds } \\
\hline Microorg. & & $\mathbf{2 a}$ & $\mathbf{2 b}$ & $\mathbf{2 c}$ & $\mathbf{2 d}$ & $\mathbf{2 e}$ & $\mathbf{2 f}$ & Ref $^{*}$ \\
\hline \multirow{3}{*}{ A } & MIC & 0.5 & 2 & 2 & 0.5 & 0.5 & 0.5 & 1 \\
\cline { 2 - 10 } & MBC & 1 & 2 & 2 & 0.5 & $>2$ & 0.5 & 1 \\
\hline \multirow{2}{*}{ B } & MIC & 1 & 2 & 2 & 1 & 1 & 1 & 1 \\
\cline { 2 - 10 } & MBC & 2 & 2 & 2 & 1 & $>2$ & 2 & 1 \\
\hline \multirow{3}{*}{ C } & MIC & 1 & 2 & 2 & 1 & 1 & 0.5 & 1 \\
\cline { 2 - 10 } & MBC & $>2$ & 2 & 2 & $>2$ & $>2$ & $>2$ & 1 \\
\hline \multirow{2}{*}{ D } & MIC & 2 & 2 & 1 & 1 & 0.5 & 0.5 & 1 \\
\cline { 2 - 10 } & MBC & 2 & 2 & $>2$ & 1 & 2 & 1 & 1 \\
\hline \multirow{2}{*}{ E } & MIC & 2 & 2 & 1 & 1 & 0.5 & 1 & 1 \\
\cline { 2 - 10 } & MBC & $>2$ & 2 & 1 & $>2$ & $>2$ & $>2$ & 1 \\
\hline \multirow{2}{*}{ F } & MIC & 2 & 2 & 1 & 1 & 0.5 & 1 & 1 \\
\cline { 2 - 10 } & MBC & $>2$ & $>2$ & 1 & $>2$ & $>2$ & $>2$ & 1 \\
\hline \multirow{2}{*}{ G } & MIC & 2 & 2 & 1 & 1 & 0.5 & 1 & 1 \\
\cline { 2 - 10 } & MBC & $>2$ & $>2$ & 1 & $>2$ & $>2$ & $>2$ & $>1$ \\
\hline
\end{tabular}

* Reference : Nitrofurazone; MIC: Minimum inhibition concentration; MBC: Minimum bactericidal concentration

A : Bacillus cereus ATCC 14579; B : Escherichia coli ATCC 25922; C : Enterococcus faecalis ATCC 29212; D : Staphylococcus epidermidis ATCC 12228; E : Staphylococcus aureus ATCC 25923; F : Salmonella typhi ATCC 14028; G : Listeria monocytogenes ATCC 7644

\section{REFERENCES:}

1. Soni, J.N.; Soman, S.S. Synthesis and Antimicrobial Evaluation of Amide Derivatives of Benzodifuran-2-carboxylic Acid. Eur. J. Med. Chem. 2014, 75, 77-81.

2. El-Gohary, N.S.; Shaaban, M.I. Synthesis, Antimicrobial, Antiquorum-sensing, Antitumor and Cytotoxic Activities of New Series of Fused [1,3,4]thiadiazoles. Eur. J. Med. Chem. 2013, $63,185-195$.

3. http://apps.who.int/iris/bitstream/10665/112647/1/WHO_HSE_PED_AIP_2014.2_eng. pdf. Last accsessed 25 August 2016. World Health Organization. Antimicrobial resistance global report on surveillance: 2014 summary.

4. Chu, P.S.; Lopez, M.I.; Abraham, A.; El Said, K.R.; Plakas, S.M. Residue Depletion of Nitrofuran Drugs and Their Tissue-Bound Metabolites in Channel Catfish (Ictalurus punctatus) after Oral Dosing. J. Agric. Food Chem. 2008, 56, 8030-8034. 
5. Dodd, M.C.; Stillman, W.B. The In Vitro Bacteriostatic Action of Some Simple Furan Derivatives. J. Pharmacol. Exp. Ther. 1944, 82, 11-18.

6. Korolkovas, A. Essentials of Medicinal Chemistry. 2nd ed. Wiley: New York, 1988.

7. Chung, MC.; Bosquesi, P.L.; dos Santos, J.L. A Prodrug Approach to Improve the PhysicoChemical Properties and Decrease the Genotoxicity of Nitro Compounds. Curr. Pharm. Design 2011, 17, 3515-3526.

8. Aguirre, G.; Boiani, L.; Cerecetto, H.; Fernandez, M.; Gonzalez, M.; Denicola, A.; Otero, L.; Gambino, D.; Rigol, C.; Olea-Azard, C.; Faundez, M. In Vitro Activity and Mechanism of Action Against the Protozoan Parasite Trypanosoma cruzi of 5-Nitrofuryl Containing Thiosemicarbazones. Bioorg. Med. Chem. 2004, 12; 4885-4893.

9. Bot, C.; Hall, B.S.; Álvarez, G.; Di Maio, R.; González, M.; Cerecetto, H.; Wilkinson, S.R.. Evaluating 5-Nitrofurans as Trypanocidal Agents. Antimicrob Agents Chemother. 2013, 57, 1638-1647.

10. Mohammadhosseini, N.; Saniee, P.; Ghamaripour, A.; Aryapour, H.; Afshar, F.; Edraki, N.; Siavoshi, F.; Foroumadi, A.; Shafiee, A. Synthesis and Biological Evaluation of Novel Benzyl Piperazine Derivatives of 5-(5-Nitroaryl)-1,3,4-thiadiazoles as Anti-Helicobacter pylori Agents. Daru 2013, 8, 21-66.

11. Behrouzi-Fardmoghadam, M.; Poorrajab, F.; Ardestani, S.K.; Emami, S.; Shafieea, A.; Foroumadi, A. Synthesis and In Vitro Anti-leishmanial Activity of 1-[5-(5-Nitrofuran-2-yl)-1,3,4thiadiazol-2-yl]- and 1-[5-(5-Nitrothiophen-2-yl)-1,3,4-thiadiazol-2-yl]-4-aroylpiperazines. Bioorg. Med. Chem. 2008, 15, 4509-4515.

12. Foroumadi, A.; Pournourmohammadi, S.; Soltani, F.; Asgharian-Rezaee, M.; Dabiri, S.; Kharazmi, A.; Shafiee, A. Synthesis and In Vitro Leishmanicidal Activity of 2-(5-Nitro-2-furyl) and 2-(5-Nitro-2-thienyl)-5-Substituted-1,3,4-thiadiazoles. Bioorg. Med. Chem. Lett. 2005, 15, 1983-1985.

13. Aguirre, G.; Boiani, M.; Cabrera, E.; Cerecetto, H.; Di Maio, R.; González, M.; Denicola, A.; Sant'anna, C.M.; Barreiro, E.J. New Potent 5-Nitrofuryl Derivatives as Inhibitors of Trypanosoma cruzi Growth. 3D-QSAR (CoMFA) Studies. Eur. J. Med. Chem. 2006, 41, 457-466.

14. Otero, L.; Vieites, M.; Boiani, L.; Denicola, A.; Rigol, C.; Opazo, L.; Olea-Azar, C.; Maya, J.D.; Morello, A., Krauth-Siegel, R.L.; Piro, O.E., Castellano, E.; González, M.; Gambino, D.; Cerecetto, H. Novel Antitrypanosomal Agents Based on Palladium Nitrofurylthiosemicarbazone Complexes: DNA and Redox Metabolism as Potential Therapeutic Targets. J. Med. Chem. 2006, 49, 3322-3331.

15. Liu, W.; Tao, C.; Tang, L.; Li, J.; Jin, Y.; Zhao, Y.; Hu, H. A Convenient and Efficient Synthesis of Heteroaromatic Hydrazone Derivatives via Cyclization of Thiosemicarbazone with a-Bromoacetophenone. J. Heterocyclic Chem. 2011, 48, 361-364.

16. D. Nardi, E. Massarani, A. Tajana, L. Degen, M.J. Magistretti. Antibacterial Nitrofuran Derivatives. I. 5-Nitro-2-furaldehyde semicarbazone and thiosemicarbazones. J. Med. Chem., 1967, 10 (4), pp 530-533.

17. Donovick, R.; Pansy, F.; Stryker, G.; Bernstein, J. The Chemotherapy of Experimental Tuberculosis. I. The In Vitro Activity of Thiosemicarbazides, Thiosemicarbazones, and Related Compouns. J. Bacteriol. 1950, 59, 667-674.

18. Otero, L.; Maya, J.D.; Morello, A.; Rigol, C.; Barriga, G.; Rodríguez, J.; Folch, C.; Norambuena, E.; González, M.; Olea Azar, C.; Cerecetto, H.; Gambino, D. Insight into the Bioreduc- 
tive Mode of Action of Antitrypanosomal 5- Nitrofuryl Containing Thiosemicarbazones. Med. Chem. 2008, 4, 11-17.

19. Secci, D.; Bizzarri, B.; Bolasco, A.; Carradori, S.; D’Ascenzio, M.; Rivanera, D.; Mari, E.; Polletta, L.; Zicari, A. Synthesis, Anti-Candida Activity, and Cytotoxicity of New (4-(4-iodophenyl) thiazol-2-yl)hydrazine Derivatives. Eur. J. Med. Chem. 2012, 53, 246-253.

20. Chimenti, F.; Bizzarri, B.; Bolasco, A.; Secci, D.; Chimenti, P.; Granese, A.; Carradori, S.; D’Ascenzio, M.; Lilli, D.; Rivanera, D. Synthesis and Biological Evaluation of Novel 2,4-Disubstituted-1,3-thiazoles as Anti-Candida spp. Agents. Eur. J. Med. Chem. 2011, 46, 378-382.

21. Hassan, A.A.; Ibrahim, Y.R.; El-Sheref, E.M.; Abdel-Aziz, M.; Bräse, S.; Nieger, M. Synthesis and Antibacterial Activity of 4-Aryl-2-(1-substituted ethylidene)thiazoles. Arch. Pharm. Chem. Life Sci. 2013, 346, 562-570.

22. Carradori, S.; Rotili, D.; De Monte, C.; Lenoci, A.; D’Ascenzio, M.; Rodriguez, V.; Filetici, P.; Miceli, M.; Nebbioso, A.; Altucci, L.; Secci, D.; Mai, A.. Evaluation of A Large Library of (Thiazol-2-yl)hydrazones and Analogues as Histone Acetyltransferase Inhibitors: Enzyme and Cellular Studies. Eur. J. Med. Chem. 2014, 80, 569-578.

23. Clinical and Laboratory Standards Institute (CLSI), Methods for Dilution Antimicrobial Susceptibility Tests for Bacteria That Grow Aerobically, Approved Standard M7-A7, CLSI,Wayne, Pa, USA, 7th edition, 2006.

24. Lukevics, E.; Jansone, D.; Rubina, K.; Abele, E.; Germane, S.; Leite, L.; Shymanska, M.; Popelis, J. Neurotropic Activity of Aldehyde and Ketone Thiosemicarbazones with A Heterocyclic Component. Eur. J. Med. Chem. 1995, 30, 983-988.

(Received 25 August 2016; accepted o8 September 2016) 
52 | Acta Pharmaceutica Sciencia. Vol. 54 No. 1, 2016 